\title{
Fluctuations in blood biomarkers of head trauma in NCAA football athletes over the course of a season
}

\author{
Jonathan M. Oliver, PhD, ${ }^{1-3}$ Anthony J. Anzalone, MS, ${ }^{1-3}$ Jason D. Stone, MS,1-3 \\ Stephanie M. Turner, MS,,-3 Damond Blueitt, MD,1-3 J. Craig Garrison, PhD, ${ }^{1-3}$ \\ Andrew T. Askow, BS, ${ }^{1,4}$ Joel A. Luedke, MS, ${ }^{5}$ and Andrew R. Jagim, PhD ${ }^{4}$
}

${ }^{1}$ Sports Concussion Research Group, Department of Kinesiology, Texas Christian University; ${ }^{2}$ Texas Health Sports Medicine Concussion Center; and ${ }^{3}$ Texas Health Sports Medicine, Fort Worth, Texas; and ${ }^{4}$ Exercise \& Sport Science Department and ${ }^{5}$ Athletics Department, University of Wisconsin—La Crosse, Wisconsin

OBJECTIVE Repetitive subconcussive head trauma is a consequence of participation in contact sports and may be linked to neurodegenerative diseases. The degree of neurological injury caused by subconcussive head trauma is not easily detectible, and this injury does not induce readily identifiable clinical signs or symptoms. Recent advancements in immunoassays make possible the detection and quantification of blood biomarkers linked to head trauma. Identification of a blood biomarker that can identify the extent of neurological injury associated with subconcussive head trauma may provide an objective measure for informed decisions concerning cumulative exposure to subconcussive head trauma. The purpose of the current study was to examine changes in the blood biomarkers of subconcussive head trauma over the course of an American football season.

METHODS Thirty-five National Collegiate Athletic Association (NCAA) American football athletes underwent blood sampling throughout the course of a football season. Serial samples were obtained throughout the 2016 season, during which the number and magnitude of head impacts changed. Blood samples were analyzed for plasma concentrations of tau and serum concentrations of neurofilament light polypeptide (NF-L). Athletes were grouped based on their starter status, because athletes identified as starters are known to sustain a greater number of impacts. Between-group differences and time-course differences were assessed.

RESULTS In nonstarters, plasma concentrations of tau decreased over the course of the season, with lower values observed in starters; this resulted in a lower area under the curve (AUC) (starters: $416.78 \pm 129.17 \mathrm{pg} / \mathrm{ml} / \mathrm{day}$; nonstarters: $520.84 \pm 163.19 \mathrm{pg} / \mathrm{ml} / \mathrm{day} ; p=0.050$ ). Plasma concentrations of tau could not be used to discern between starters and nonstarters. In contrast, serum concentrations of NF-L increased throughout the season as head impacts accumulated, specifically in those athletes categorized as starters. The higher serum concentrations of NF-L observed in starters resulted in a larger AUC (starters: $1605.03 \pm 655.09 \mathrm{pg} / \mathrm{ml} / \mathrm{day}$; nonstarters: $1067.29 \pm 272.33 \mathrm{pg} / \mathrm{ml} / \mathrm{day} ; \mathrm{p}=0.007$ ). The AUC of the receiver operating characteristic curve analyses displayed fair to modest accuracy to identify athletes who were starters with the use of serum NF-L following periods of repetitive impacts.

CONCLUSIONS The different patterns observed in serum NF-L and plasma tau concentrations provide preliminary evidence for the use of blood biomarkers to detect the neurological injury associated with repetitive subconcussive head trauma. Although further investigation is necessary, such findings might lay the foundation for the further development of an objective measure for the detection of neurological injury caused by subconcussive head trauma.

https://thejns.org/doi/abs/10.3171/2017.12.JNS172035

KEYWORDS sports-related head trauma; sports-related concussion; mild traumatic brain injury; blood biomarkers of head trauma 
$\mathrm{T}$ HROUGHOUT the course of a season, American football athletes are routinely exposed to head impacts that vary in magnitude and frequency. ${ }^{9,11}$ Most of these incidents do not result in a concussion diagnosis and are thus known as subconcussive events. Although there is little evidence that subconcussive head impacts result in any discernible clinical effects, ${ }^{4}$ there is some indication that the accumulation of subconcussive impacts over the course of a competitive season is linked to detectable neurophysiological and neuropsychological changes. ${ }^{1,7,38}$ Preliminary research suggests that an accumulation of subconcussive impacts associated with long-term participation in contact sports may be implicated in the development of neurodegenerative disorders such as chronic traumatic encephalopathy (CTE). ${ }^{22}$

Diffuse microscopic damage to white matter tracts, more commonly known as diffuse axonal injury (DAI), is a pathological consequence of traumatic brain injury (TBI), ${ }^{18,35}$ mild TBI (mTBI), ${ }^{5,37}$ and repetitive subconcussive head trauma. ${ }^{2,3}$ In less severe injuries, DAI is detectable only by advanced neuroimaging techniques. DAI appears to be linked to the number and magnitude of subconcussive head impacts sustained by American football athletes at both the high school and collegiate levels. . $^{32,19,20,36,38}$ While advanced imaging techniques may detect DAI that is acquired via repetitive subconcussive impacts, it is improbable that team medical staff at most levels have the resources to perform routine diffusion imaging throughout the competitive season.

Recent advancements in ultrasensitive single-molecule array techniques make possible the detection of blood biomarkers sensitive to brain injury. ${ }^{17}$ In contrast to advanced imaging techniques, routine blood sampling and quantification of blood biomarkers of brain injury may prove to be an easier, less time-intensive, and more cost-effective method for detecting damage due to repetitive subconcussive impacts sustained by contact-sport athletes. The central nervous system-specific proteins tau and neurofilament light polypeptide (NF-L) are fluid biomarkers of head trauma that have been well studied. ${ }^{40}$ Principally found in axons, NF-L is a cytoskeletal constituent that functions to provide structural support to the neuron. ${ }^{35}$ Tau, which is also enriched in axons, is critical for microtubule assembly and stability. ${ }^{10}$ Neurological insult disrupts the integrity of the axonal cytoskeleton, a feature of DAI,, 1735 and a recent case-series investigation of patients with TBI found that the serum concentration of NF-L may reflect the degree of $\mathrm{DAI}^{18}$ - evidence of the potential usefulness of blood biomarkers in the detection of brain injury. Early investigations indicate that peripheral concentrations of tau and NF-L are acutely elevated following both $\mathrm{TBI}^{6,18,32}$ and $\mathrm{mTBI} ;{ }^{15,27,33}$ however, there are less substantive patterns of biomarker changes following subconcussive head trauma. Initial reports suggest that tau may be a more sensitive indicator in the acute phase following injury, during which the effects of the injury are clinically discernible ${ }^{15,33}$ (leading to a diagnosis of concussion), while NF-L may be a more sensitive indicator of subconcussive head trauma and may remain elevated for several days following the most recent exposure. ${ }^{28-30,34}$

Given evidence that subconcussive head trauma associ- ated with long-term contact sport participation is linked to neurodegenerative disease, ${ }^{21,22}$ and that subconcussive head trauma does not induce readily identifiable clinical signs or symptoms, ${ }^{4}$ a search for a valid and feasible method for detecting the neurological damage of subconcussive head trauma is warranted. Feasible methods for detecting the neurological damage of subconcussive head trauma may ultimately allow for the determination of injury thresholds, which would provide an objective measure for informed decisions concerning cumulative exposure. As an example, elevations in biomarker concentration above a certain yet-to-be determined threshold may reveal the extent of DAI that has occurred as a result of subconcussive impacts. Such information could prove invaluable for team medical staff charged with the health and safety of athletes. Clinicians would effectively have a tool for assisting in decisions regarding the removal of athletes from play to dampen cumulative neurological damage. Most importantly, the utility of such biomarkers may aide in mitigating the potential long-term neurological consequences of repetitive sports-related head trauma.

To that end, in the current study we sought to examine the effect of routine subconcussive head impacts on peripheral tau and NF-L in American football athletes. In addition, we sought to examine the potential clinical utility of both biomarkers. We hypothesized that the serum concentration of NF-L would increase over the course of a season-specifically in starters, those athletes known to sustain a greater number of head impacts than other team players - and that the plasma concentration of tau would remain stable over the course of a season and not differ between starters and nonstarters. Based on the prior hypothesis, we further hypothesized that NF-L would provide better diagnostic utility than tau in identifying those athletes who sustain a greater number of impacts.

\section{Methods \\ Participants}

This study was conducted according to the principles and guidelines set forth by the Declaration of Helsinki. All procedures involving humans were approved by the Institutional Review Board of Texas Christian University for the use of human subjects in research. Written consent was obtained from all participants. Volunteers were apparently healthy National Collegiate Athletic Association (NCAA) Division III American football athletes who had been cleared to participate by the team physician. Athletes were excluded from the study if they sustained a concussion or any other injury that inhibited their ability to participate in regularly scheduled practice or competition at any time during the study.

\section{Study Design}

A prospective longitudinal cross-sectional study design was used to examine changes in the biomarkers of head trauma in college athletes over the course of a season of Division III American football. In addition, given that some football athletes-identified as starters-are known to be exposed to a greater number of head impacts due to their participation in a greater number of repetitions (plays) 
in each game, ${ }^{8}$ the biomarkers of interest were compared between starters and nonstarters. The coaching staff was consulted to determine the status of starters, and starters were defined as those athletes who are participants in the first or second team, are first or second on the depth chart, and are those who perform most repetitions (approximately 20-40+) during competition (game play).

Nonfasting blood samples were obtained at specific times during the 2016 football season. A timeline associated with sampling is provided in Fig. 1. The first sample was obtained at time 1 (T1), following a 14-week noncontact period during which athletes only participated in strength and conditioning. Thereafter, samples were obtained coincident with times during which changes in the number and/or magnitude of head impacts likely occurred. The second sample (T2) was obtained 15 days following T1, at the conclusion of fall camp. Fall camp lasted 13 days and consisted of 4 strength and conditioning sessions as well as 16 total practices. Fall camp practices began as noncontact sessions and progressed to full-contact practices with full equipment until the conclusion of the camp. In the NCAA, fall camp is associated with the highest concentration of impacts for participating athletes, regardless of their starter status, due to the concentrated number of full-contact practices occurring during a short time. Following fall camp, samples were obtained every 21 days, on a Monday. The third sample (T3) was obtained 21 days after fall camp concluded; this occurred after the beginning of the season and following a bye week (a week in which the team has no game), making it 9 days after the previous game and approximately 72 hours after the previous full-contact practice. Samples 4 and 5 (T4 and T5) were collected on Mondays, approximately 36 hours following a Saturday game. Samples 6 and 7 (T6 and T7) were collected after the conclusion of the competitive season, 10 and 33 days, respectively, after the last game. Throughout the competitive season, athletes participated in full-gear practices on Tuesdays and Wednesdays, followed by a partial-gear (helmets and shoulder pads) practice on Thursdays and no practice on Sunday, Monday, or Friday. The week preceding T3 was the only exception to this schedule; that week full-gear practices occurred on Tuesday, Wednesday, Thursday, and Friday.

\section{Blood Collection and Analyses}

Nonfasting venous blood $(10 \mathrm{ml})$ was collected via venipuncture from the antecubital fossa region by using standard phlebotomy procedures. Serum was collected using vacutainer tubes with no additive, and plasma was collected using vacutainers that had been spray coated with $\mathrm{K}_{2}$-ethylenediaminetetraacetic acid (EDTA) (BD Diagnostics). Contents of the serum vacutainer tubes were al- lowed to clot for 30 minutes at room temperature and were subsequently centrifuged at $2000 \mathrm{~g}$ for 10 minutes at $4^{\circ} \mathrm{C}$. The $\mathrm{K}_{2}$-EDTA tubes were inverted following collection and subsequently centrifuged at $2000 \mathrm{~g}$ for 20 minutes at $4^{\circ} \mathrm{C}$ (Allegra X-15R, Beckman Coulter) within 30 minutes after collection. Following centrifugation, samples were transferred to prelabeled polypropylene vials and stored at $-80^{\circ} \mathrm{C}$ until biomarker quantification.

\section{Biomarker Quantification}

Plasma concentrations of tau (Simoa Tau 2.0 Kit) and serum concentrations of NF-L (Simoa Beta Kit) were measured using digital array technology on a Single Molecule Array (Simoa) HD-1 Analyzer, software version 1.5 (Quanterix). The same lot of kits was used to assay both tau and NF-L. Prior to the analyses, the samples were diluted 1:4, except when insufficient volume was present and a dilution of 1:5 or 1:6 was used. The concentrations of each sample at all time points $(0.061 \mathrm{pg} / \mathrm{ml}$ for tau and $0.171 \mathrm{pg} / \mathrm{ml}$ for NF-L) were greater than the lower limits of quantification. The lower limit of detection was 0.007 $\mathrm{pg} / \mathrm{ml}$ for tau and $0.050 \mathrm{pg} / \mathrm{ml}$ for NF-L. Duplicates were run with a median dose coefficient of variation of 5\% for tau and $4 \%$ for NF-L.

\section{Statistical Analyses}

Outcomes of interest were initially assessed using a repeated-measures analysis of variance with the factors time (7 levels) and starter status (2 levels). The area under the curve (AUC), calculated using the trapezoidal method, was compared between groups-starters and nonstarters-using an independent samples t-test. To determine the potential diagnostic utility of the biomarkers in relation to impacts sustained over the course of the season, a smallest magnitude of effect was defined: the baseline standard deviation among all subjects $\left(\mathrm{SD}_{\text {within }}\right)$. Calculation of the difference between each time point and the baseline was performed and identified by a binary categorical variable $(0=$ no, $1=$ yes $)$ for each participant. Chi-square analysis was then used to evaluate the statistical difference of the categorical outcome variable between starters and nonstarters. The area under the receiver operating characteristic (ROC) curve was calculated for each biomarker, tau and NF-L, in a comparison of starters and nonstarters, in order to determine whether the biomarker concentration could be used to accurately identify players by their starter status. All statistical analyses were performed using the Statistical Package for Social Sciences (SPSS, version 24.0). Graphs were created using GraphPad Prism, version 5.0 (GraphPad, Inc.). Group values are reported as means \pm SDs.
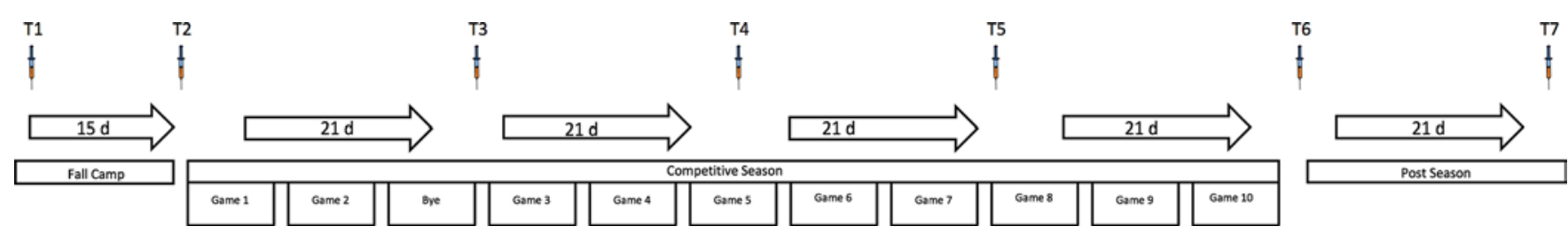

FIG. 1. Timeline associated with blood sampling. Figure is available in color online only. 


\section{Results \\ Demographics}

After excluding those athletes with injuries and/or those unable to participate in practice or competition, 35 athletes (age $21 \pm 1$ years [range 19-23 years]; height 180.9 $\pm 7.5 \mathrm{~cm}$; and weight $94.9 \pm 13.1 \mathrm{~kg}$ ) were included in the analyses. Of these, 20 were categorized as starters and the remaining 15 were categorized as nonstarters. The athletes categorized as starters were older ( $21 \pm 1$ years) than the nonstarters $(20 \pm 1$ years; $p<0.01)$. No additional differences were observed between the two groups.

\section{Plasma Concentration of Tau}

Prior to the start of fall camp, the mean plasma concentration of tau was significantly lower in starters (4.23 $\pm 1.72 \mathrm{pg} / \mathrm{ml})$ than in nonstarters $(5.89 \pm 2.64 \mathrm{pg} / \mathrm{ml} ; \mathrm{p}$ $=0.04)$. Throughout the season, the plasma tau concentration was decreased in both starters and nonstarters compared to concentrations measured before fall camp. The tau concentration returned to pre-fall camp values in starters by the first sampling after the competitive season (T6), which corresponded to 10 days after the last competition, and in nonstarters by the second sampling after the competitive season (T7) (Fig. 2). The overall lower values of tau identified in starters resulted in a lower AUC for the starters (starters: $416.78 \pm 129.17 \mathrm{pg} / \mathrm{ml}$ day; nonstarters: $520.84 \pm 163.19 \mathrm{pg} / \mathrm{ml} / \mathrm{day} ; \mathrm{p}=0.05)$.

Since an increase in the plasma concentration of tau was expected, the lack of an increase above the smallest magnitude, defined as the baseline standard deviation among all subjects $\left(\mathrm{SD}_{\text {within }}\right)$, resulted in no significant difference between starters and nonstarters when one compared the proportions of athletes with increased tau protein above the threshold (Table 1). The AUC of the ROC curve analyses demonstrated that plasma concentrations of tau failed to discern between those athletes categorized as starters and nonstarters, with the highest AUC, 0.42 (95\% CI 0.21-0.62; $\mathrm{p}=0.44$ ), observed 33 days after the last competition, at T7 (Table 2).

\section{Serum Concentrations of NF-L}

Serum concentrations of NF-L at the post-camp sampling (T2) were found to be increased above those observed before the start of fall camp (T1) in both starters and nonstarters (Fig. 3). However, in nonstarters the increase in NF-L concentrations did not reach statistical significance $(\mathrm{p}=0.59)$ and, thereafter, serum concentrations of NF-L did not differ significantly at any time point throughout the rest of the season. In contrast, the serum concentration of NF-L was elevated above pre-fall camp concentrations at several samplings throughout the course of the season in starters (Fig. 3). These elevations in serum NF-L concentrations in starters were significantly higher than concentrations in nonstarters. Additionally, the higher serum concentrations of NF-L observed in starters resulted in a larger AUC for that group (starters: 1605.03 $\pm 655.09 \mathrm{pg} / \mathrm{ml} / \mathrm{day}$; nonstarters: $1067.29 \pm 272.33 \mathrm{pg} / \mathrm{ml} /$ day; $\mathrm{p}=0.007$ ).

A higher proportion of starters were found to have an increase in NF-L that was greater than the determined

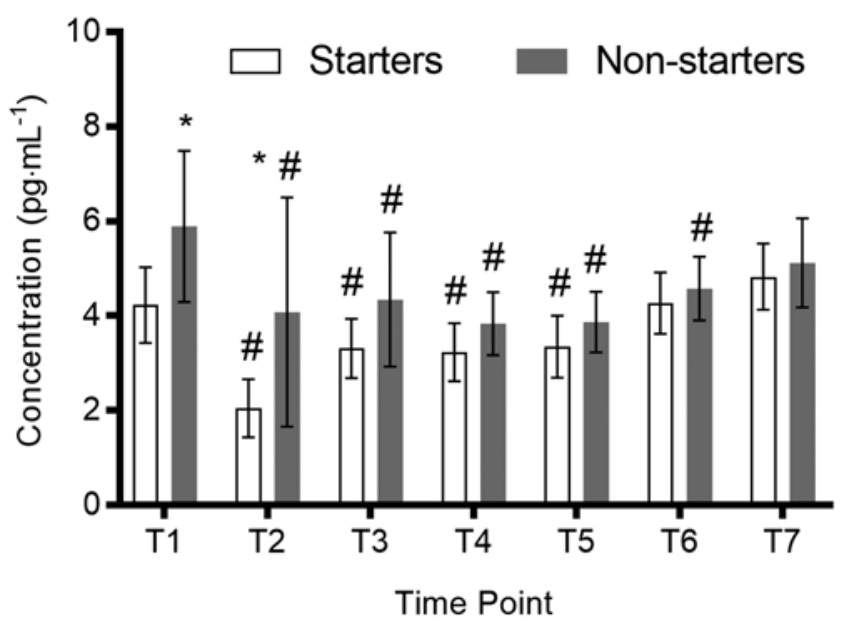

FIG. 2. Plasma concentrations of tau over the course of the season in American football athletes. Asterisk indicates significant difference by starter status ( $p \leq 0.05)$; pound sign indicates significant difference from $\mathrm{T} 1(\mathrm{p} \leq 0.05)$.

threshold at all time points (Table 1). The AUC of the ROC curve analyses showed that the serum concentration of NF-L exhibited poor accuracy prior to the beginning of the season; however, after periods of repetitive head impacts began, the concentration of NF-L exhibited fair to modest accuracy for the identification of athletes who were starters (Table 2).

\section{Discussion}

Unlike many contact sports in which subconcussive impacts are not routine, repetitive subconcussive impacts occur commonly in football. Given that these repetitive

TABLE 1. Chi-square analyses comparing the proportion of players in whom concentrations of tau or NF-L increased above the smallest magnitude of effect, defined as $S_{D_{\text {within }}}$ baseline, based on starter status

\begin{tabular}{|c|c|c|c|c|}
\hline & All Players (\%) & Nonstarters (\%) & Starters (\%) & $p$ Value \\
\hline \multicolumn{5}{|c|}{ Plasma tau } \\
\hline $\mathrm{T} 2$ & $1(2.9)$ & $1(6.7)$ & $0(0.0)$ & 0.24 \\
\hline T3 & $0(0.0)$ & $0(0.0)$ & $0(0.0)$ & - \\
\hline T4 & $0(0.0)$ & $0(0.0)$ & $0(0.0)$ & - \\
\hline T5 & $0(0.0)$ & $0(0.0)$ & $0(0.0)$ & - \\
\hline T6 & $0(0.0)$ & $0(0.0)$ & $0(0.0)$ & - \\
\hline $\mathrm{T} 7$ & $4(12.1)$ & $1(7.7)$ & $3(15.0)$ & 0.53 \\
\hline \multicolumn{5}{|c|}{ Serum NF-L } \\
\hline $\mathrm{T} 2$ & $14(40.0)$ & $3(20.0)$ & $11(55.0)$ & 0.04 \\
\hline T3 & $15(45.5)$ & $3(21.4)$ & $12(63.2)$ & 0.02 \\
\hline $\mathrm{T} 4$ & $13(37.1)$ & $3(20.0)$ & $10(50.0)$ & 0.07 \\
\hline T5 & $10(28.6)$ & $2(13.3)$ & $8(40.0)$ & 0.08 \\
\hline T6 & $11(33.3)$ & $1(7.1)$ & $10(52.6)$ & 0.01 \\
\hline $\mathrm{T} 7$ & $6(18.2)$ & $1(7.1)$ & $5(26.3)$ & 0.16 \\
\hline
\end{tabular}

See Methods for details about the conditions at each time point ( $\mathrm{T}$ ) and why those time points were chosen. 
TABLE 2. ROC AUC and corresponding statistics at each time point for tau and NF-L in starters compared with nonstarters

\begin{tabular}{|c|c|c|c|}
\hline & AUC & $95 \% \mathrm{Cl}$ & p Value \\
\hline \multicolumn{4}{|c|}{ Plasma tau concentration } \\
\hline T1 & 0.22 & $0.07-0.37$ & 0.01 \\
\hline $\mathrm{T} 2$ & 0.25 & $0.09-0.42$ & 0.02 \\
\hline T3 & 0.33 & $0.12-0.54$ & 0.10 \\
\hline T4 & 0.34 & $0.16-0.52$ & 0.11 \\
\hline T5 & 0.38 & $0.19-0.57$ & 0.23 \\
\hline T6 & 0.39 & $0.19-0.59$ & 0.10 \\
\hline $\mathrm{T} 7$ & 0.42 & $0.22-0.62$ & 0.44 \\
\hline \multicolumn{4}{|c|}{ Serum NF-L concentration } \\
\hline T1 & 0.57 & $0.38-0.76$ & 0.48 \\
\hline $\mathrm{T} 2$ & 0.69 & $0.52-0.87$ & 0.05 \\
\hline T3 & 0.76 & $0.59-0.92$ & 0.01 \\
\hline T4 & 0.67 & $0.49-0.85$ & 0.09 \\
\hline T5 & 0.72 & $0.55-0.89$ & 0.03 \\
\hline T6 & 0.75 & $0.58-0.92$ & 0.02 \\
\hline T7 & 0.71 & $0.53-0.89$ & 0.04 \\
\hline
\end{tabular}

See Methods for details about the conditions at each time point $(\mathrm{T})$ and why those time points were chosen.

impacts result in neurological changes, even in the absence of significant clinical effects, ${ }^{4}$ identification and quantification of damage associated with subconcussive head trauma via an easily obtainable blood sample may be useful for monitoring players at risk and determining ways to reduce the potential long-term consequences associated with repetitive head trauma. In the current study, the plasma concentration of tau decreased over the course of the season in football athletes. In contrast, the serum concentration of NF-L increased over the course of the season, particularly in starters. Furthermore, the serum concentration of NF-L demonstrated moderate accuracy for identification of starters throughout the season, as the number of subconcussive impacts increased. The results of this study suggest that circulating concentrations of tau and NF-L differ, and that further research is warranted before either biomarker can become a practical tool with which sports medicine professionals may monitor the physiological effects of subconcussive head trauma.

Microtubules are stabilized by a variety of proteins, one of which is tau..$^{10}$ The presence of tau in biological fluid samples may indicate a dissociation from microtubules, followed by their destabilization and the eventual development of neurofibrillary tangles. ${ }^{40}$ Neurofibrillary tangles are a pathological characteristic of CTE, a tauopathy attributed to repetitive mTBI. ${ }^{23}$ Following a sportsrelated concussion, elevations in plasma tau are observed throughout the first 12 hours ${ }^{15,33}$ and have been reported to persist for up to 72 hours after the injury in some athletes. However, extended elevations of plasma tau ( $\geq 72$ hours) are observed only in those athletes with more severe injuries, as indicated by a prolonged duration of recovery. ${ }^{15}$ In the current study, the timing of sampling, 36-72 hours after competition or practice, may have contributed to the

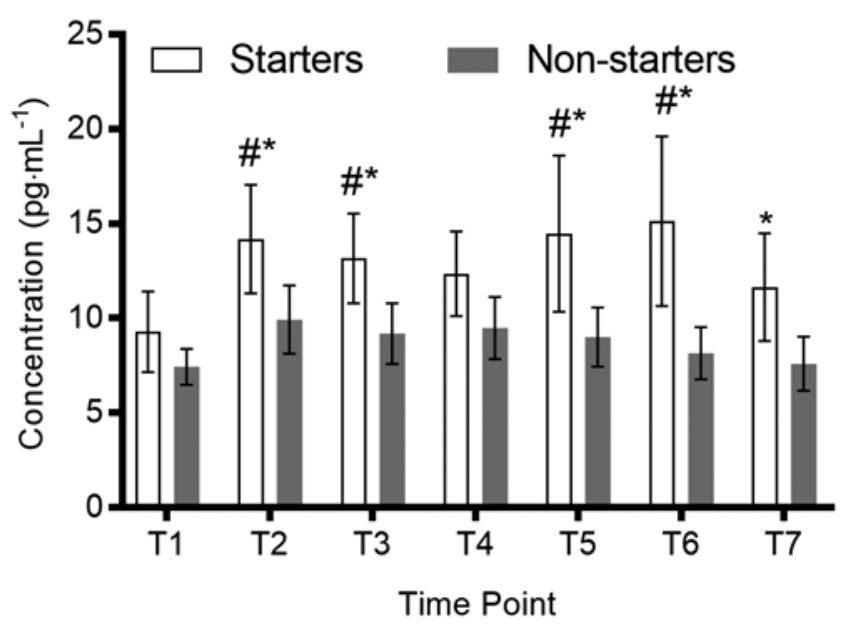

FIG. 3. Serum concentrations of NF-L over the course of the season in American football athletes. Asterisk indicates significant difference by starter status $(p \leq 0.05)$; pound sign indicates significant difference from $\mathrm{T} 1$ ( $p \leq 0.05)$.

absence of any observable plasma tau increase over the course of the season. Though elevations in plasma tau were reported in Olympic boxers 1-6 days following a bout during which no concussion occurred, ${ }^{27}$ no changes in plasma tau were observed in ice hockey players following a game. ${ }^{33}$ Similar to boxers, ice hockey and football players are routinely exposed to head impacts. Head impacts occur at a greater rate in athletes engaged in football routinely than in athletes participating in ice hockey ${ }^{25}$ which is why we hypothesized that tau concentrations would increase over the course of the season in those football athletes categorized as starters. Biomechanical analyses of impacts that occur in boxing and football have shown that differences exist: rotational acceleration is greater in boxing and the head injury criterion (the mathematic quantitative assessment of head injury risk as a result of impact parameters) and translational acceleration are greater in football. ${ }^{39}$ It must also be noted that while the methods of detection in the current study and the previous study in Olympic boxers are similar, the assay results differed such that although concentration elevations were observed in both studies, the mean tau concentrations at all time points examined were lower in the Olympic boxers than those reported in the football players in the current study.

Physical activity status may also affect the plasma concentration of tau. A recent study reported increases in tau 6 hours postinjury in college athletes who had sustained a concussion, although the same athletes had significantly lower concentrations 24 and 72 hours postinjury compared to athlete controls who had not sustained a concussion. ${ }^{15}$ The reductions in plasma tau observed at the later time points (i.e., 24 and 72 hours postinjury) may be related to the cessation of physical activity that occurs following a concussion diagnosis. Higher levels of plasma tau 6 months following injury, when physical activity has resumed, may support the potential role for physical activity on plasma concentrations of tau. Thus, sampling that occurred 36-72 hours after the last bout of physical activity may, at least partially, explain the lack of an observable increase in tau. 
A unique finding in the current study is that prior to the start of the season, coincident with a 14-week period of noncontact (T1), the plasma tau level was higher in nonstarters than in starters. In contrast, we recently reported that following a 9-week period of noncontact (T1), starters had higher plasma tau levels than nonstarters. ${ }^{28}$ There is a notable difference between our earlier study and the current one: in the former study the T1 sampling was conducted before formal summer strength and conditioning sessions, whereas in the current study the T1 sampling was conducted just before the start of the season and did not account for summer strength and conditioning. Nonetheless, the difference may be explained by differences in previous physical activity levels as well as by possible differences in exposure to cumulative head impacts, which were not accounted for in the current study. As such, future studies should aim to control for previous amounts of physical activity as well as cumulative exposure to head impacts so that differences that may exist between starters and nonstarters can be better characterized. While further study is warranted to examine the effects of both cumulative head impact exposure and physical activity on plasma tau levels, the results of the current study are largely in agreement with those of previous work $^{30,34}$ and suggest that tau may not be the optimal choice for detection of damage associated with subconcussive impacts sustained by football athletes.

In contrast to plasma tau, serum NF-L was observed to be elevated over the course of the season in those athletes categorized as starters. Serum NF-L appeared to be useful for differentiating between athletes known to sustain a greater number of impact exposures (starters) and those not known to do so (nonstarters). Neurofilaments, the primary intermediate filaments in neurons, are major components of the axonal cytoskeleton ${ }^{13,31}$ and are relatively long-lived proteins (the estimated half-life of NF-L is approximately 3 weeks). ${ }^{24}$ Animal models indicate that significant changes occur to neurofilaments as a result of DAI (for a review see Siedler et al. ${ }^{35}$ ), and alterations to neurofilaments may precede microtubule destabilization when axons are placed under a mechanical load (such as in traumatic injury). ${ }^{14}$ This may be why NF-L has been suggested to be a more sensitive and specific biomarker of neuroaxonal injury than other recently identified biomarkers. ${ }^{26,34}$ Moreover, a recent case-series investigation identified a link between serum NF-L and changes on diffusion tensor imaging (DTI) studies following TBI, which is suggestive that serum NF-L may indeed be a reliable marker of DAI ${ }^{18}$ Elevations in NF-L obtained from cerebrospinal fluid (CSF) were first reported in Olympic boxers and were correlated with the number of sustained concussive or subconcussive head impacts. ${ }^{26}$ Those early findings were recently confirmed in another study examining NF-L in the same population; in that study CSF concentrations of NF-L were also highly correlated with serum concentrations of NF-L $(r=0.86 ; p<0.01){ }^{34}$

Findings in the current study indicate that, as exposures to subconcussive head trauma accumulate throughout a competitive season, serum NF-L levels increase and remain elevated until participation in the sport ceases. Future work should aim at the further identification of the utility of biomarkers that are cost-effective and easier to obtain than advanced imaging studies. Furthermore, the identification of a biomarker that is reflective of the neurological damage accrued via subconcussive head traumabut one that does not need to be obtained immediately following head trauma exposure and is not sensitive only to clinically discernible injuries or factors such as physical activity (as in the case of tau) - is critical.

In considering the utility of such a biomarker, let us look at collegiate football athletes as an example. A team medical staff's ability to obtain and properly process an athlete's blood sample within the first 24 hours following a game may be difficult due to travel and accommodation factors, and it will certainly vary based on the level of the program (e.g., Division I vs Division III). In the current study, we sought to use a more feasible window of time following the last competition or practice (approximately 36-72 hours) to investigate the potential utility of NF-L as a biomarker of subconcussive head trauma. Similar findings were recently reported in a smaller cohort of NCAA American football athletes: serum NF-L was elevated over the course of a season, whereas plasma tau decreased during the same time period. ${ }^{28-30}$ In the current study, we used a larger player sample and our findings are in agreement with our previous findings and those in boxers. ${ }^{34}$ The athletes in the current study were NCAA Division III athletes; those in our previous studies were NCAA Division I players. Our findings suggest that, irrespective of the level of play, which is associated with differences in the magnitude of head impacts, the subconcussive impacts sustained by football athletes may result in some level of axonal damage, as identified by increases in serum NF-L. The difference in the proportions of starters and nonstarters, in whom an increase in NF-L above the threshold was identified, further demonstrates the potential for quantification of NF-L to be used as an objective measure for clinical decision making regarding cumulative head impact exposure. The observable patterns of increase and decrease in this biomarker that were observed in starters in the current study suggest that NF-L may be sensitive to damage resulting from subconcussive impacts and, perhaps more importantly, to the cumulative effects of subconcussive impacts.

\section{Limitations of the Study}

There are, of course, limitations to our study. First, a much larger study is necessary to develop the most appropriate threshold for NF-L or other biological markers associated with subconcussive impacts. Still, the current study is the largest to date, providing new information related to the effect of a season of football on biological markers of head trauma and expanding on previous research focused on NF-L and tau. ${ }^{28,30}$ An additional limitation lies in the lack of objective measures of the number and magnitude of head impacts sustained by athletes in this study; however, a number of studies have demonstrated that the magnitude of impact is not necessarily associated with the likelihood of a concussion or the development of symptoms. ${ }^{16} \mathrm{~A}$ lack of advanced neuroimaging data (e.g., MR-DTI) limits the ability to determine whether the elevated concentrations that we observed, specifically in NF-L, were a result of neurophysiological damage; nonetheless, the recent link 
reported between serum NF-L and DTI supports the potential utility for NF-L as a biomarker.

\section{Conclusions}

Given that repetitive subconcussive impacts may produce neurological impairments that are greater in severity than those associated with a single incident, ${ }^{7}$ identification of a biological marker(s) that can be used to clinically manage repetitive subconcussive head trauma would be beneficial in improving long-term brain health. Tau and NF-L have shown utility as markers of acute injury; however, there are limited data on the utility of these biomarkers in subconcussive impacts. The results of the current study suggest that both tau and NF-L should be further explored as biomarkers of subconcussive damage due to the differences observed in starters, athletes known to sustain a greater number of subconcussive impacts. Although an examination of tau levels may prove useful following diagnosed concussive injuries, serum NF-L appears to increase over the course of the season in football athletes known to sustain a greater number of impacts. Further, determination of serum concentrations of NF-L demonstrates moderate accuracy in the identification of those athletes categorized as starters. Together, these results suggest that further work should be conducted to verify whether the quantification of serum NF-L provides a sensitive and reliable biological marker of subconcussive head trauma. Such advances would lay the groundwork for the eventual development of clinical tools to help reduce the deleterious effects of repetitive subconcussive impacts. As opposed to biomechanical measurements, which vary markedly regarding the threshold for a clinical diagnosis of concussion, thus limiting the ability to define subconcussive events, biological markers such as NF-L may reflect the actual damage resulting from subconcussive impacts and thus provide a more objective tool for clinical decision making.

\section{References}

1. Abbas K, Shenk TE, Poole VN, Breedlove EL, Leverenz LJ, Nauman EA, et al: Alteration of default mode network in high school football athletes due to repetitive subconcussive mild traumatic brain injury: a resting-state functional magnetic resonance imaging study. Brain Connect 5:91-101, 2015

2. Bazarian JJ, Zhu T, Blyth B, Borrino A, Zhong J: Subjectspecific changes in brain white matter on diffusion tensor imaging after sports-related concussion. Magn Reson Imaging 30:171-180, 2012

3. Bazarian JJ, Zhu T, Zhong J, Janigro D, Rozen E, Roberts A, et al: Persistent, long-term cerebral white matter changes after sports-related repetitive head impacts. PLoS One 9:e94734, 2014

4. Belanger HG, Vanderploeg RD, McAllister T: Subconcussive blows to the head: a formative review of short-term clinical outcomes. J Head Trauma Rehabil 31:159-166, 2016

5. Blumbergs PC, Scott G, Manavis J, Wainwright H, Simpson DA, McLean AJ: Staining of amyloid precursor protein to study axonal damage in mild head injury. Lancet 344:10551056, 1994

6. Bogoslovsky T, Wilson D, Chen Y, Hanlon D, Gill J, Jeromin A, et al: Increases of plasma levels of glial fibrillary acidic protein, tau, and amyloid $\beta$ up to 90 days after traumatic brain injury. J Neurotrauma 34:66-73, 2017
7. Breedlove EL, Robinson M, Talavage TM, Morigaki KE, Yoruk U, O'Keefe K, et al: Biomechanical correlates of symptomatic and asymptomatic neurophysiological impairment in high school football. J Biomech 45:12651272,2012

8. Broglio SP, Eckner JT, Martini D, Sosnoff JJ, Kutcher JS, Randolph C: Cumulative head impact burden in high school football. J Neurotrauma 28:2069-2078, 2011

9. Broglio SP, Schnebel B, Sosnoff JJ, Shin S, Fend X, He X, et al: Biomechanical properties of concussions in high school football. Med Sci Sports Exerc 42:2064-2071, 2010

10. Cleveland DW, Hwo SY, Kirschner MW: Purification of tau, a microtubule-associated protein that induces assembly of microtubules from purified tubulin. J Mol Biol 116:207-225, 1977

11. Crisco JJ, Fiore R, Beckwith JG, Chu JJ, Brolinson PG, Duma S, et al: Frequency and location of head impact exposures in individual collegiate football players. J Athl Train 45:549-559, 2010

12. Davenport EM, Whitlow CT, Urban JE, Espeland MA, Jung Y, Rosenbaum DA, et al: Abnormal white matter integrity related to head impact exposure in a season of high school varsity football. J Neurotrauma 31:1617-1624, 2014

13. Fletcher DA, Mullins RD: Cell mechanics and the cytoskeleton. Nature 463:485-492, 2010

14. Fournier AJ, Rajbhandari L, Shrestha S, Venkatesan A, Ramesh KT: In vitro and in situ visualization of cytoskeletal deformation under load: traumatic axonal injury. FASEB J 28:5277-5287, 2014

15. Gill J, Merchant-Borna K, Jeromin A, Livingston W, Bazarian J: Acute plasma tau relates to prolonged return to play after concussion. Neurology 88:595-602, 2017

16. Guskiewicz KM, Mihalik JP: Biomechanics of sport concussion: quest for the elusive injury threshold. Exerc Sport Sci Rev 39:4-11, 2011

17. Ling H, Hardy J, Zetterberg H: Neurological consequences of traumatic brain injuries in sports. Mol Cell Neurosci 66 (Pt B):114-122, 2015

18. Ljungqvist J, Zetterberg H, Mitsis M, Blennow K, Skoglund T: Serum neurofilament light protein as a marker for diffuse axonal injury: results from a case series study. J Neurotrauma 34:1124-1127, 2017

19. Mayinger, MC, Merchant-Borna, K, Hufschmidt, J, Muehlmann, M, Weir, IR, Rauchmann, BS, et al: White matter alterations in college football players: a longitudinal diffusion tensor imaging study. Brain Imaging Behav [epub ahead of print], 2017

20. McAllister TW, Ford JC, Flashman LA, Maerlender A, Greenwald RM, Beckwith JG, et al: Effect of head impacts on diffusivity measures in a cohort of collegiate contact sport athletes. Neurology 82:63-69, 2014

21. McKee AC, Cantu RC, Nowinski CJ, Hedley-Whyte ET, Gavett $\mathrm{BE}$, Budson $\mathrm{AE}$, et al: Chronic traumatic encephalopathy in athletes: progressive tauopathy after repetitive head injury. J Neuropathol Exp Neurol 68:709-735, 2009

22. McKee AC, Daneshvar DH, Alvarez VE, Stein TD: The neuropathology of sport. Acta Neuropathol 127:29-51, 2014

23. McKee AC, Stern RA, Nowinski CJ, Stein TD, Alvarez VE, Daneshvar DH, et al: The spectrum of disease in chronic traumatic encephalopathy. Brain 136:43-64, 2013

24. Millecamps S, Gowing G, Corti O, Mallet J, Julien JP: Conditional NF-L transgene expression in mice for in vivo analysis of turnover and transport rate of neurofilaments. $\mathbf{J}$ Neurosci 27:4947-4956, 2007

25. Naunheim RS, Standeven J, Richter C, Lewis LM: Comparison of impact data in hockey, football, and soccer. J Trauma 48:938-941, 2000

26. Neselius S, Brisby H, Theodorsson A, Blennow K, Zetterberg H, Marcusson J: CSF-biomarkers in Olympic boxing: 
diagnosis and effects of repetitive head trauma. PLoS One 7:e33606, 2012

27. Neselius S, Zetterberg H, Blennow K, Randall J, Wilson D, Marcusson J, et al: Olympic boxing is associated with elevated levels of the neuronal protein tau in plasma. Brain Inj 27:425-433, 2013

28. Oliver JM, Jones MT, Anzalone AJ, Kirk KM, Gable DA, Repshas JT, et al: A season of American football is not associated with changes in plasma tau. J Neurotrauma 34:32953300, 2017

29. Oliver JM, Jones MT, Kirk KM, Gable DA, Repshas JT, Johnson TA, et al: Effect of docosahexaenoic acid on a biomarker of head trauma in American football. Med Sci Sports Exerc 48:974-982, 2016

30. Oliver JM, Jones MT, Kirk KM, Gable DA, Repshas JT, Johnson TA, et al: Serum neurofilament light in American football athletes over the course of a season. J Neurotrauma 33:1784-1789, 2016

31. Perrot R, Berges R, Bocquet A, Eyer J: Review of the multiple aspects of neurofilament functions, and their possible contribution to neurodegeneration. Mol Neurobiol 38:27-65, 2008

32. Shahim P, Gren M, Liman V, Andreasson U, Norgren N, Tegner Y, et al: Serum neurofilament light protein predicts clinical outcome in traumatic brain injury. Sci Rep 6:36791, 2016

33. Shahim P, Tegner Y, Wilson DH, Randall J, Skillbäck T, Pazooki D, et al: Blood biomarkers for brain injury in concussed professional ice hockey players. JAMA Neurol 71:684-692, 2014

34. Shahim P, Zetterberg H, Tegner Y, Blennow K: Serum neurofilament light as a biomarker for mild traumatic brain injury in contact sports. Neurology 88:1788-1794, 2017

35. Siedler DG, Chuah MI, Kirkcaldie MT, Vickers JC, King AE: Diffuse axonal injury in brain trauma: insights from alterations in neurofilaments. Front Cell Neurosci 8:429, 2014

36. Slobounov SM, Walter A, Breiter HC, Zhu DC, Bai X, Bream $\mathrm{T}$, et al: The effect of repetitive subconcussive collisions on brain integrity in collegiate football players over a single football season: a multi-modal neuroimaging study. Neuroimage Clin 14:708-718, 2017

37. Spain A, Daumas S, Lifshitz J, Rhodes J, Andrews PJ, Horsburgh K, et al: Mild fluid percussion injury in mice produces evolving selective axonal pathology and cognitive deficits relevant to human brain injury. J Neurotrauma 27:1429-1438, 2010

38. Talavage TM, Nauman EA, Breedlove EL, Yoruk U, Dye AE, Morigaki KE, et al: Functionally-detected cognitive impairment in high school football players without clinicallydiagnosed concussion. J Neurotrauma 31:327-338, 2014

39. Viano DC, Casson IR, Pellman EJ, Bir CA, Zhang L, Sherman DC, et al: Concussion in professional football: comparison with boxing head impacts - part 10. Neurosurgery 57:1154-1172, 2005

40. Zetterberg H, Smith DH, Blennow K: Biomarkers of mild traumatic brain injury in cerebrospinal fluid and blood. Nat Rev Neurol 9:201-210, 2013

\section{Disclosures}

This study was funded in part by a grant from STRUCT Nutrition.

\section{Author Contributions}

Conception and design: all authors. Acquisition of data: Anzalone, Stone, Turner, Askow, Luedke, Jagim. Analysis and interpretation of data: Oliver, Anzalone, Stone, Blueitt, Garrison. Drafting the article: Oliver, Anzalone, Stone, Garrison, Jagim. Critically revising the article: all authors. Reviewed submitted version of manuscript: all authors. Statistical analysis: Oliver. Administrative/technical/material support: Anzalone, Blueitt, Luedke, Jagim. Study supervision: Oliver, Anzalone, Askow, Luedke, Jagim.

\section{Correspondence}

Jonathan M. Oliver: Texas Christian University, Fort Worth, TX. jonathan.oliver@tcu.edu. 